\title{
Efficient synthesis of functionalized spiro[imidazolidine-2-thione- oxindoles] via catalyst-free domino Mannich cyclization
}

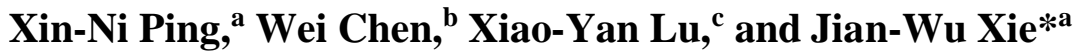 \\ ${ }^{a}$ Department of Chemistry and Life Science, Zhejiang Normal University, \\ Jinhua 321004, P. R. China \\ ${ }^{b}$ Zhejiang Pharmaceutical College, Ningbo 315100, P. R. China \\ ${ }^{c}$ Ningbo No. 2 Hospital, Ningbo 315010, P. R. China \\ E-mail: xiejw@zjnu.cn
}

DOI: https://doi.org/10.24820/ark.5550190.p009.932

\begin{abstract}
An efficient protocol has been developed for the synthesis of spiro[imidazolidine-2-thioneoxindole] derivatives with multi-functionalized groups via catalyst-free domino reaction of by domino Mannich/cyclization of 3-isothiocyanato oxindoles and bis(arylmethylidene)hydrazines. The domino reaction can proceed smoothly in an environmentally benign conditions and provides pure functionalized spiro[imidazolidine-2-thione-oxindole] derivatives with excellent diastereoselectivity in moderate to excellent yield.
\end{abstract}

Keywords: Domino reaction, spirooxindoles, bis(arylmethylidene)hydrazines, Mannich reaction, heterocycles

\section{Introduction}

The development of efficient methods for the synthesis of new spirocyclic skeleton has been a topic of great relevance in organic synthesis because this class of compounds have a wide range of excellent biological activities. The spirocyclic oxindole derivatives are recognized as attractive synthetic targets because of their prevalence in numerous natural products and pharmaceutical agents as well as useful intermediates for the easy access of a variety of heterocyclic compounds by rearrangement reaction due to their steric strain associated with the quaternary carbon. ${ }^{1-4}$

Recently, 3-isothiocyanato oxindoles have been used as the most attractive substrates in catalytic cascade Michael/cyclization reactions, ${ }^{5-9}$ Mannich/cyclization reactions ${ }^{10-11}$ Aldol/cyclization reactions ${ }^{12-13}$ and [3+2] cyclization ${ }^{14-18}$ for the synthesis of the highly functionalized spirocyclic oxindole derivatives. 


\section{Results and Discussion}

Due to their varied biological activities and important precursors, design and development of a straightforward and "greener" approach for the construction of new functionalized spirooxindoles is still interesting. Very recently, we synthesized a series of 3-isothiocyanato oxindoles and successfully used them as nucleophiles for asymmetric synthesis of a range of enantioenriched multi-functionalized tetracyclic spirooxindoles with multiple stereocenters. ${ }^{19}$ Based on this achievement and our recent successes in the development of new methodologies for the construction functionalized heterocycles using domino reactions, we envisioned that spiro[imidazolidine-2-thione-oxindole] derivatives with multi-functionalized groups could be synthesized by domino Mannich/cyclization of 3-isothiocyanato oxindoles and bis(arylmethylidene)hydrazines under mild conditions (Eq. 2, Scheme 1).

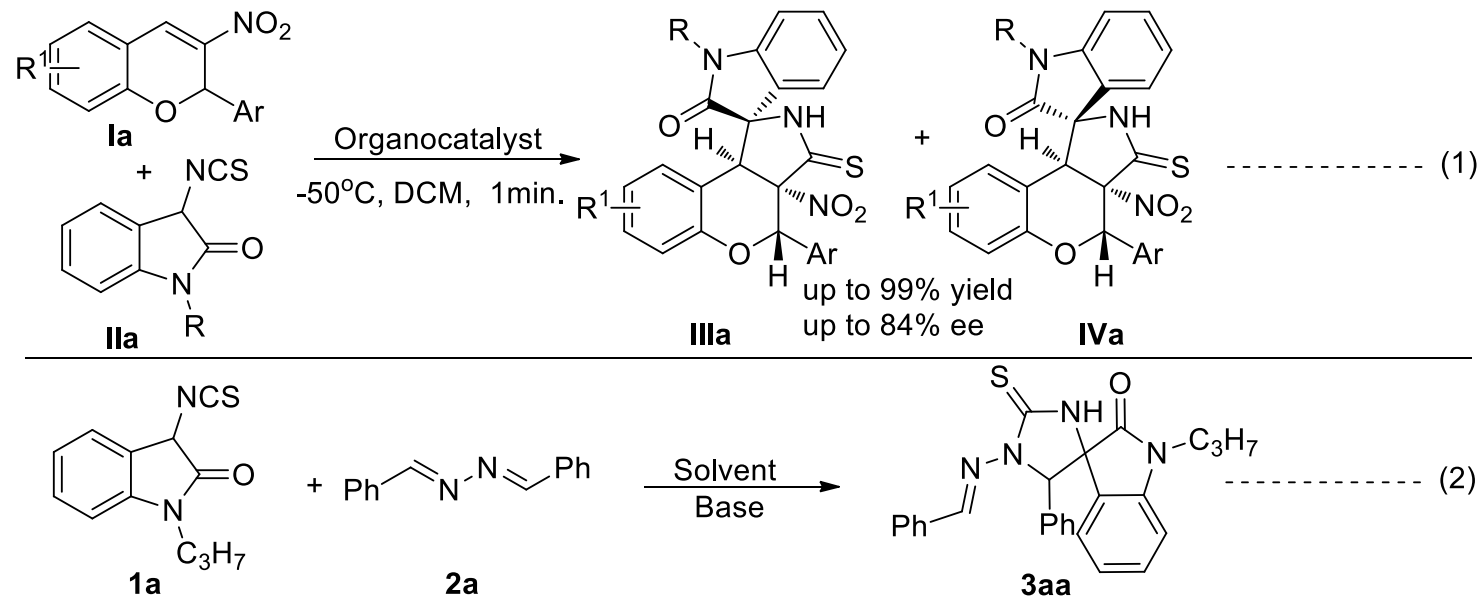

Scheme 1. Synthetic approach to dihydrofurans via domino Michael addition-alkylation reaction.

Table 1. Screening for the optimum conditions ${ }^{\mathrm{a}}$

\begin{tabular}{ccccc}
\hline Entry & Base & Solvent & ${\text { Yield } \%^{\mathrm{b}}}^{\mathrm{c}}$ & $\mathrm{Dr}^{\mathrm{c}}$ \\
\hline 1 & $\mathrm{KOH}$ & $\mathrm{THF}$ & 23 & $>99: 1$ \\
2 & $\mathrm{~K}_{2} \mathrm{CO}_{3}$ & THF & 67 & $>99: 1$ \\
3 & $\mathrm{KOAc}$ & THF & 70 & $>99: 1$ \\
4 & $\mathrm{DABCO}$ & THF & 79 & $>99: 1$ \\
5 & $\mathrm{DABCO}$ & toluene & 54 & $>99: 1$ \\
6 & $\mathrm{DABCO}$ & acetone & 86 & $>99: 1$ \\
7 & $\mathrm{DABCO}$ & ethanol & 95 & $>99: 1$ \\
8 & $\mathrm{DABCO}$ & $\mathrm{H}_{2} \mathrm{O}$ & 47 & $>99: 1$ \\
$10^{\mathrm{d}}$ & - & ethanol & 86 & $>99: 1$ \\
\hline
\end{tabular}

${ }^{\text {a }}$ Otherwise noted, reactions performed with $0.10 \mathrm{mmol}$ of $\mathbf{1 a}, 0.10 \mathrm{mmol}$ of $2 \mathrm{a}, 20 \mathrm{~mol} \%$ of base in $1 \mathrm{~mL}$ solvent at room temperature for $1 \mathrm{~h} .{ }^{\mathrm{b}}$ Isolated yield. ${ }^{\mathrm{c}}$ Determined by ${ }^{1} \mathrm{H}$ NMR. ${ }^{\mathrm{d}} 0.5 \mathrm{~h}$. 
We first studied the domino Mannich/cyclization of 3-isothiocyanato oxindole 1a with 1,4dibenzalhydrazine 2a. Several organic solvents and bases were screened for the domino reaction and the representative results are shown in Table 1 . When the strong base $\mathrm{KOH}$ was used as the catalyst, the domino Mannich/cyclization of 3-isothiocyanato oxindole 1a with 1,4dibenzalhydrazine 2a became complicated and low yield was isolated (Table 1, entry 1, 23\% yield). Then the weaker bases, such as $\mathrm{K}_{2} \mathrm{CO}_{3}$ and KOAc was screened for the domino reaction, and good yields were obtained (Table 1, entries 2-3). The organic base DABCO (1,4diazabicyclo[2.2.2] octane) was used as the catalyst, the yield of the desired product was higher than those by using $\mathrm{KOH}, \mathrm{K}_{2} \mathrm{CO}_{3}$ and $\mathrm{KOAc}$ as the catalysts. Subsequently, the effects of solvent on the reactivity were investigated using $\mathrm{DABCO}$ as the catalyst. The reaction in a polar aprotic solvent such as THF and toluene afforded only 3aa with somewhat lower yields (Table 1, entries 4 and 5). On the contrary, the desired product 3aa was formed in high yield, when the reaction was carried out in a protic solvent, EtOH. Among the solvents screened, the use of ethanol gave the excellent result (Table 1, entry 7, 95\% yield). To our delight, the domino reaction proceeded smoothly to provide desired product spiro[imidazolidine-2-thione-oxindoles] 3aa with high yield under catalyst-free conditions when ethanol was used as solvent (Table 1, entry 9, 94\% yield). Maybe the hydrogen atom of ethanol could activate bis(arylmethylidene)hydrazine $\mathbf{2 a}$ by the hydrogen bond between ethanol and nitrogen atom of the bis(arylmethylidene)hydrazine 2a. In addition, the yield was reduced when the reaction time was shortened (able 1, entry 10).

Consequently, an array of bis(arylmethylidene)hydrazines 2 were explored in the reactions with 3-isothiocyanato oxindoles 1 to establish the general utility of this new methodology. The catalyst-free domino Mannich/cyclization was generally conducted in ethanol for $1 \mathrm{~h}$ at room temperature. As summarised in Table 2, all the products were obtained with excellent diastereoselectivities (>99:1). The electronic characteristics of bis(arylmethylidene)hydrazines 2 seemed to have significant effects on the yield except of 2e. High yields were obtained for a diversity of bis(arylmethylidene)hydrazines $\mathbf{2}$ bearing electron-withdrawing groups on the aromatic ring (Table 2, entries 2-5 and 9-11). On the contrary, bis(arylmethylidene)hydrazines 2 bearing electron-donating groups on the aromatic ring tended to decrease the reactivity and only moderate yields were obtained (Table 2, entries 6-7 and 12-13). In addition, the position of substituent on aryl ring of bis(arylmethylidene)hydrazines 2 had little influence on the yields, spiro[imidazolidine-2-thione-oxindole] derivatives were also obtained with high yields. Bis(arylmethylidene)hydrazines $\mathbf{2 h}$ with furanyl substitution exhibited a good reactivity, and the good results (78\% yield) were still obtained (Table 2, entry 8). The stereochemistries of spiro[imidazolidine-2-thione-oxindole] derivatives $\mathbf{3}$ were established unambiguously by X-ray analysis of 3aa (Figure 1). The relative configuration was confirmed as illustrated by the ORTEP diagram depicted in Figure 1. 
Table 2. Reaction between 3-isothiocyanato oxindoles $\mathbf{1}$ and bis(arylmethylidene)hydrazines $\mathbf{2}^{\mathrm{a}}$

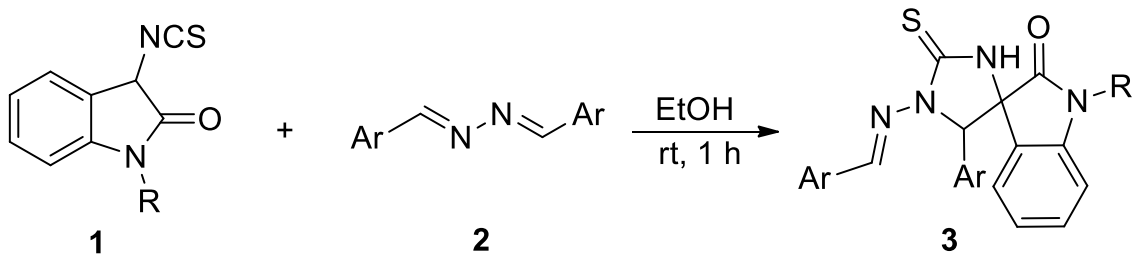

\begin{tabular}{llccc}
\hline \multicolumn{1}{c}{ Entry } & $\mathrm{R}(\mathbf{1})$ & $\mathrm{Ar}(\mathbf{2})$ & Yield (3) \% & $\mathrm{Dr}^{\mathrm{c}}$ \\
\hline 1 & $n-\mathrm{C}_{3} \mathrm{H}_{7}(\mathbf{1 a})$ & $\mathrm{C}_{6} \mathrm{H}_{5}(\mathbf{2 a})$ & $94(\mathbf{3 a a})$ & $>99: 1$ \\
2 & $n-\mathrm{C}_{3} \mathrm{H}_{7}(\mathbf{1 a})$ & $p-\mathrm{ClC}_{6} \mathrm{H}_{4}(\mathbf{2 b})$ & $90(\mathbf{3 a b})$ & $>99: 1$ \\
3 & $n-\mathrm{C}_{3} \mathrm{H}_{7}(\mathbf{1 a})$ & $m-\mathrm{ClC}_{6} \mathrm{H}_{4}(\mathbf{2 c})$ & $89(\mathbf{3 a c})$ & $>99: 1$ \\
4 & $n-\mathrm{C}_{3} \mathrm{H}_{7}(\mathbf{1 a})$ & $p-\mathrm{BrC}_{6} \mathrm{H}_{4}(\mathbf{2 d})$ & $84(\mathbf{3 a d})$ & $>99: 1$ \\
5 & $n-\mathrm{C}_{3} \mathrm{H}_{7}(\mathbf{1 a})$ & $p-\mathrm{CF}_{3} \mathrm{C}_{6} \mathrm{H}_{4}(\mathbf{2 e})$ & $68(\mathbf{3 a e})$ & $>99: 1$ \\
6 & $n-\mathrm{C}_{3} \mathrm{H}_{7}-(\mathbf{1 a})$ & $p-\mathrm{MeOC}_{6} \mathrm{H}_{4}(\mathbf{2 f})$ & $65(\mathbf{3 a f})$ & $>99: 1$ \\
7 & $n-\mathrm{C}_{3} \mathrm{H}_{7}(\mathbf{1 a})$ & $m-\mathrm{MeOC}_{6} \mathrm{H}_{4}(\mathbf{2 g})$ & $60(\mathbf{3 a g})$ & $>99: 1$ \\
8 & $n-\mathrm{C}_{3} \mathrm{H}_{7}(\mathbf{1 a})$ & $2-\mathrm{Furyl}_{(\mathbf{1 h})}$ & $78(\mathbf{3 a h})$ & $>99: 1$ \\
9 & $\mathrm{Me}(\mathbf{1 b})$ & $o-\mathrm{ClC}_{6} \mathrm{H}_{4}(\mathbf{1 i})$ & $90(\mathbf{3 b i})$ & $>99: 1$ \\
10 & $\mathrm{Me}(\mathbf{1 b})$ & $p-\mathrm{NO}_{2} \mathrm{C}_{6} \mathrm{H}_{4}(\mathbf{1 j})$ & $89(\mathbf{3 b j})$ & $>99: 1$ \\
11 & $\mathrm{Me}(\mathbf{1 b})$ & $m-\mathrm{NO}_{2} \mathrm{C}_{6} \mathrm{H}_{4}(\mathbf{2 k})$ & $85(\mathbf{3 b k})$ & $>99: 1$ \\
12 & $\mathrm{Me}(\mathbf{1 b})$ & $o-\mathrm{MeC}_{6} \mathrm{H}_{4}(\mathbf{2})$ & $68(\mathbf{3 b l})$ & $>99: 1$ \\
13 & $\mathrm{Me}(\mathbf{1 b})$ & $m-\mathrm{MeC}_{6} \mathrm{H}_{4}(\mathbf{2 m})$ & $65(\mathbf{3 b m})$ & $>99: 1$ \\
\hline
\end{tabular}

${ }^{\text {a }}$ Otherwise noted, reactions performed with $0.10 \mathrm{mmol}$ of $\mathbf{1}, 0.10 \mathrm{mmol}$ of 2 in $1 \mathrm{~mL}$ ethanol at room temperature for $1 \mathrm{~h} .{ }^{\mathrm{b}}$ Isolated yield. ${ }^{\mathrm{c}}$ Determined by ${ }^{1} \mathrm{H}$ NMR.
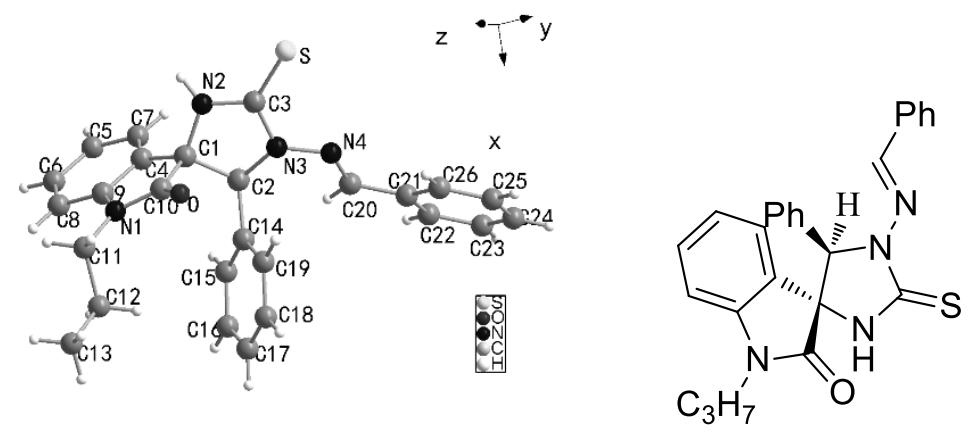

Figure 1. X-ray crystal structure of (E)-1-(benzylideneamino)-5-phenyl-1'-propyl-2thioxospiro[imidazolidine-4,3'-indolin]-2'-one 3aa.

On the basis of the experimental observations, a plausible mechanistic were proposed, as shown in Scheme 2. The reaction proceeds faster in ethanol than that in other organic solvents without catalyst, since hydrogen atom of ethanol could activate bis(arylmethylidene)hydrazines 2 
by the hydrogen bond between ethanol and nitrogen atom of the bis(arylmethylidene)hydrazines $\mathbf{2}$, increasing electrophilic property of the $a$-carbon. In addition, the hydrogen bond between ethanol and nitrogen atom of the 3-isothiocyanato oxindoles 1 increases the electrophilic property of 3-isothiocyanato oxindoles 1. Thus, a domino Mannich/cyclization between 3-isothiocyanato oxindoles $\mathbf{1}$ and bis(arylmethylidene)hydrazines $\mathbf{2}$ proceeded smoothly using ethanol as solvent.

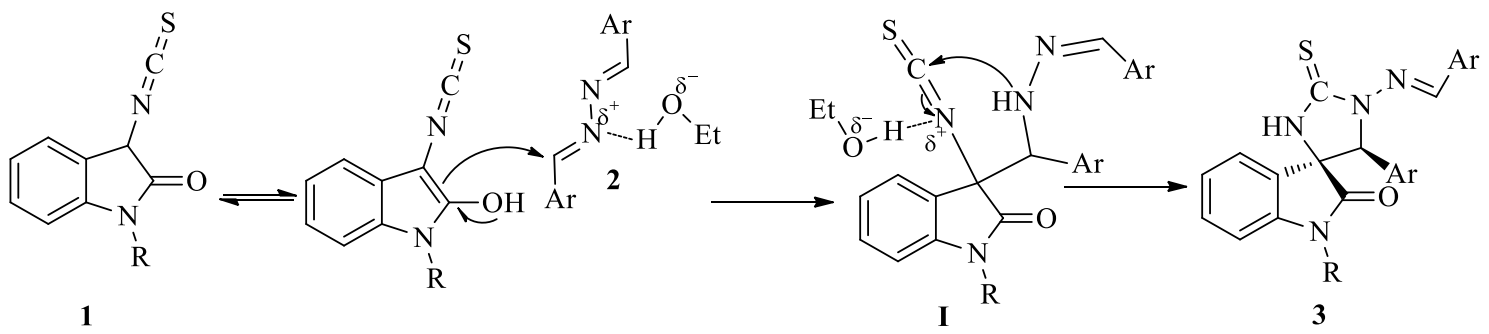

Scheme 2. Proposed mechanism of domino Mannich/cyclyzation of 3-isothiocyanato oxindoles 1 and bis(arylmethylidene)hydrazines 2.

\section{Conclusions}

An efficient method for the synthesis of functionalized spiro[imidazolidine-2-thione-oxindole] derivatives $\mathbf{3}$ by catalyst-free domino Mannich/cyclyzation of 3-isothiocyanato oxindoles $\mathbf{1}$ and bis(arylmethylidene)hydrazines $\mathbf{2}$ has been explored. The catalyst-free domino reaction can proceed smoothly in an environmentally benign conditions and provides pure functionalized spiro[imidazolidine-2-thione-oxindole] derivatives with excellent diastereoselectivities in moderate to excellent yields. This novel methodology should be of great interest for pharmaceutical synthesis because of the mild reaction conditions.

\section{Experimental Section}

General. All reactants were commercially available and used without further purification. All melting points were uncorrected. ${ }^{1} \mathrm{H}$ NMR and ${ }^{13} \mathrm{C}$ NMR spectra were measured on a $600 \mathrm{MHz}$ Bruker spectrometer $\left({ }^{1} \mathrm{H} 600 \mathrm{MHz},{ }^{13} \mathrm{C} 150 \mathrm{MHz}\right)$, using $\mathrm{CDCl}_{3}$ as the solvent with tetramethylsilane (TMS) as the internal standard at room temperature. Chemical shifts are given in $\delta$ relative to TMS, the coupling constants $J$ are given in Hz. Column chromatography was performed using EM Silica gel 60 (300-400 mesh).

General procedure for the reaction between 3 -isothiocyanato oxindoles 1 and bis(arylmethylidene)hydrazines. A mixture of 3-isothiocyanato oxindole 1a $(0.10 \mathrm{mmol})$ and 1,4-dibenzalhydrazine $\mathbf{2 a}(0.10 \mathrm{mmol})$ was stirred in ethanol $(1 \mathrm{~mL})$ at room temperature for $1 \mathrm{~h}$. 
After that, flash chromatography on silica gel (30\% DCM/petroleum ether) gave 3aa (44 mg, $93 \%$ yield) as pale yellow solid.

(E)-1-(Benzylideneamino)-5-phenyl-1'-propyl-2-thioxospiro[imidazolidine-4,3'-indolin]-2' one (3aa). Solid, mp 218-220 ${ }^{\circ} \mathrm{C} ;{ }^{1} \mathrm{H}$ NMR (600 MHz, $\left.\mathrm{CDCl}_{3}\right) \delta 7.97$ (s, 1H), $7.72(\mathrm{~d}, J 7.3 \mathrm{~Hz}$, 1H), $7.60-7.54(\mathrm{~m}, 3 \mathrm{H}), 7.40(\mathrm{t}, J 7.7 \mathrm{~Hz}, 1 \mathrm{H}), 7.34-7.26(\mathrm{~m}, 3 \mathrm{H}), 7.22-7.17(\mathrm{~m}, 3 \mathrm{H}), 6.97$ (d, $J 7.3 \mathrm{~Hz}, 2 \mathrm{H}), 6.75(\mathrm{~d}, J 7.9 \mathrm{~Hz}, 1 \mathrm{H}), 5.57(\mathrm{~s}, 1 \mathrm{H}), 3.51-3.44(\mathrm{~m}, 1 \mathrm{H}), 3.04-2.98(\mathrm{~m}, 1 \mathrm{H}), 1.17$ $-1.09(\mathrm{~m}, 1 \mathrm{H}), 1.01-0.93(\mathrm{~m}, 1 \mathrm{H}), 0.54(\mathrm{t}, J 7.4 \mathrm{~Hz}, 3 \mathrm{H}) .{ }^{13} \mathrm{C} \mathrm{NMR}\left(150 \mathrm{MHz}, \mathrm{CDCl}_{3}\right) \delta 183.5$, 172.1, 151.1, 143.6, 133.7, 131.3, 131.0, 130.4, 129.0, 128.5, 127.8, 127.1, 126.6, 124.8, 123.4, 108.8, 74.1, 68.6, 41.7, 20.3, 11.1. ESI-HRMS: calcd for $\mathrm{C}_{26} \mathrm{H}_{24} \mathrm{~N}_{4} \mathrm{OS}+\mathrm{H} 441.1749$, found 441.1740. Crystal data for 3aa. $\mathrm{C}_{26} \mathrm{H}_{24} \mathrm{~N}_{4} \mathrm{OS}$ (440.55), (CCDC number: XXX), Monoclinic, $\mathrm{P} 2(1) / \mathrm{c} ; \mathrm{a}=13.1311(3) \AA$, alpha $=90$ deg. $\mathrm{b}=22.3023(4) \AA$, beta $=104.5990(10)$ deg. $\mathrm{c}=$ 8.2940(2) $\AA$, gamma = 90 deg. $U=2350.51(9) \AA^{3}, Z=30, T=296(2) \mathrm{K}$, absorption coefficient $0.163 \mathrm{~mm}^{-1}$, reflections collected 78909 , unique 5419 [R(int $\left.)=0.0435\right]$, refinement by Full-matrix least-squares on $F^{2}$, data/ restraints/ parameters 5419 / 0 / 290, goodness-of-fit on $F^{2}=1.067$, final $R$ indices $[I>2 \operatorname{sigma}(I)] \mathrm{R} 1=0.0573, \mathrm{wR} 2=0.1391, R$ indices (all data) $\mathrm{R} 1=0.0734, \mathrm{wR} 2=$ 0.1518 , largest diff. peak and hole 0.422 and -0.394 e. $\AA^{-3}$.

(E)-1-((4-Chlorobenzylidene)amino)-5-(4-chlorophenyl)-1'-propyl-2-thioxospiro-[imidazolidine-4,3'-indolin]-2'-one (3ab). Solid, mp 176-178 ${ }^{\circ} \mathrm{C}$; ${ }^{1} \mathrm{H}$ NMR (600 MHz, DMSO) $\delta 9.83$ (s, 1H), $7.81(\mathrm{~s}, 1 \mathrm{H}), 7.70(\mathrm{~d}, J 7.3 \mathrm{~Hz}, 1 \mathrm{H}), 7.58(\mathrm{~d}, J 8.5 \mathrm{~Hz}, 2 \mathrm{H}), 7.47-7.41(\mathrm{~m}, 3 \mathrm{H}), 7.32(\mathrm{~d}, J$ $8.5 \mathrm{~Hz}, 2 \mathrm{H}), 7.21(\mathrm{t}, J 7.5 \mathrm{~Hz}, 1 \mathrm{H}), 7.03(\mathrm{~d}, J 7.9 \mathrm{~Hz}, 1 \mathrm{H}), 6.89(\mathrm{~d}, J 8.4 \mathrm{~Hz}, 2 \mathrm{H}), 5.90(\mathrm{~s}, 1 \mathrm{H})$, $3.22-3.16(\mathrm{~m}, 1 \mathrm{H}), 1.17-1.11(\mathrm{~m}, 2 \mathrm{H}), 1.09-1.02(\mathrm{~m}, 1 \mathrm{H}), 0.51(\mathrm{t}, J 7.4 \mathrm{~Hz}, 3 \mathrm{H}) .{ }^{13} \mathrm{C} \mathrm{NMR}$ (150 MHz, DMSO) $\delta 182.9,172.2,147.5,143.7,135.1,133.8,133.2,131.2,131.0,129.5,129.1$, 128.8, 127.2, 125.2, 123.5, 109.5, 71.2, 68.4, 41.3, 20.5, 11.1. ESI-HRMS: calcd for $\mathrm{C}_{26} \mathrm{H}_{22} \mathrm{C}_{12} \mathrm{~N}_{4} \mathrm{OS}+\mathrm{H} 509.0970$, found 509.0966.

(E)-1-((3-Chlorobenzylidene)amino)-5-(3-chlorophenyl)-1'-propyl-2-thioxospiro[imidazolidine-4,3'-indolin]-2'-one (3ac). Solid, mp 174-175 ${ }^{\circ} \mathrm{C}$; ${ }^{1} \mathrm{H}$ NMR (600 MHz, DMSO) $\delta 9.91$ (s, 1H), $7.81(\mathrm{~s}, 1 \mathrm{H}), 7.70(\mathrm{~d}, J 7.1 \mathrm{~Hz}, 1 \mathrm{H}), 7.64(\mathrm{~s}, 1 \mathrm{H}), 7.50(\mathrm{~d}, J 7.3 \mathrm{~Hz}, 1 \mathrm{H}), 7.47-7.40(\mathrm{~m}, 3 \mathrm{H})$, $7.34(\mathrm{~d}, J 7.7 \mathrm{~Hz}, 1 \mathrm{H}), 7.29(\mathrm{t}, J 7.3 \mathrm{~Hz}, 1 \mathrm{H}), 7.22(\mathrm{t}, J 7.1 \mathrm{~Hz}, 1 \mathrm{H}), 7.06(\mathrm{~d}, J 7.7 \mathrm{~Hz}, 1 \mathrm{H}), 6.86$ $(\mathrm{s}, 2 \mathrm{H}), 5.90(\mathrm{~s}, 1 \mathrm{H}), 1.18-1.10(\mathrm{~m}, 2 \mathrm{H}), 1.10-1.02(\mathrm{~m}, 1 \mathrm{H}), 0.88-0.82(\mathrm{~m}, 1 \mathrm{H}), 0.54(\mathrm{t}, J 6.5$ $\mathrm{Hz}, 3 \mathrm{H}) .{ }^{13} \mathrm{C}$ NMR $(150 \mathrm{MHz}, \mathrm{DMSO}) \delta 182.9,172.1,146.9,143.6,136.5,134.3,134.0,133.4$, 131.3, 130.7, 130.3, 129.1, 127.2, 127.0, 126.6, 126.4, 126.2, 125.2, 123.6, 109.6, 71.0, 68.4, 41.3, 20.59, 11.2. ESI-HRMS: calcd for $\mathrm{C}_{26} \mathrm{H}_{22} \mathrm{C}_{12} \mathrm{~N}_{4} \mathrm{OS}+\mathrm{H} 509.0970$, found 509.0979.

(E)-1-((4-bromobenzylidene)amino)-5-(4-bromophenyl)-1'-propyl-2-thioxospiro[imidazolidine-4,3'-indolin]-2'-one (3ad). Solid, mp 194-195 ${ }^{\circ} \mathrm{C}$; ${ }^{1} \mathrm{H}$ NMR (600 MHz, DMSO) $\delta 9.84$ (s, 1H), $7.80(\mathrm{~s}, 1 \mathrm{H}), 7.71(\mathrm{~d}, J 7.4 \mathrm{~Hz}, 1 \mathrm{H}), 7.60(\mathrm{~d}, J 8.5,1.8 \mathrm{~Hz}, 2 \mathrm{H}), 7.52(\mathrm{~d}, J 8.5 \mathrm{~Hz}, 2 \mathrm{H}), 7.48$ $-7.41(\mathrm{~m}, 3 \mathrm{H}), 7.22$ (t, J 7.5 Hz, 1H), $7.05(\mathrm{~d}, J 7.9 \mathrm{~Hz}, 1 \mathrm{H}), 6.83(\mathrm{~d}, J 8.4 \mathrm{~Hz}, 2 \mathrm{H}), 5.89$ (s, 1H), $3.25-3.18(\mathrm{~m}, 1 \mathrm{H}), 1.20-1.11(\mathrm{~m}, 2 \mathrm{H}), 1.10-1.02(\mathrm{~m}, 1 \mathrm{H}), 0.53(\mathrm{t}, J 7.4 \mathrm{~Hz}, 3 \mathrm{H}) .{ }^{13} \mathrm{C} \mathrm{NMR}$ (150 MHz, DMSO) $\delta 182.9,172.2,170.8,147.4,143.7,133.5,132.3,131.7,131.3,131.2,129.5$, 129.4, 127.2, 125.2, 123.9, 123.5, 122.5, 109.5, 71.2, 68.4, 41.3, 20.5, 11.2. ESI-HRMS: calcd for $\mathrm{C}_{26} \mathrm{H}_{22} \mathrm{Br}_{2} \mathrm{~N}_{4} \mathrm{OS}+\mathrm{H} 596.9959$, found 596.9974. 
(E)-1'-Propyl-2-thioxo-1-((4-(trifluoromethyl)benzylidene)amino)-5-(4-(trifluoromethyl)phenyl)spiro[imidazolidine-4,3'-indolin]-2' -one (3ae). Solid, mp 189-190 ${ }^{\circ} \mathrm{C} ;{ }^{1} \mathrm{H}$ NMR (600 MHz, DMSO) $\delta 9.98(\mathrm{~s}, 1 \mathrm{H}), 7.85(\mathrm{~s}, 1 \mathrm{H}), 7.80-7.73(\mathrm{~m}, 5 \mathrm{H}), 7.65(\mathrm{~d}, J 8.1 \mathrm{~Hz}, 2 \mathrm{H}), 7.46(\mathrm{t}, J$ $7.8 \mathrm{~Hz}, 1 \mathrm{H}), 7.25(\mathrm{t}, J 7.5 \mathrm{~Hz}, 1 \mathrm{H}), 7.10(\mathrm{~d}, J 7.9 \mathrm{~Hz}, 2 \mathrm{H}), 7.06(\mathrm{~d}, J 7.9 \mathrm{~Hz}, 1 \mathrm{H}), 6.07(\mathrm{~s}, 1 \mathrm{H})$, $3.21-3.16(\mathrm{~m}, 1 \mathrm{H}), 1.09-1.00(\mathrm{~m}, 1 \mathrm{H}), 0.97-0.79(\mathrm{~m}, 2 \mathrm{H}), 0.45(\mathrm{t}, J 7.4 \mathrm{~Hz}, 3 \mathrm{H}) .{ }^{13} \mathrm{C} \mathrm{NMR}$ (150 MHz, DMSO) $\delta 183.0,172.1,170.8,146.4,143.7,138.2,136.5,131.4,129.6,128.3,128.1$, 126.8, 126.1, 125.7, 125.5, 125.2, 123.7, 123.4, 109.6, 71.0, 68.5, 41.3, 20.4, 11.0. ESI-HRMS: calcd for $\mathrm{C}_{28} \mathrm{H}_{22} \mathrm{~F}_{6} \mathrm{~N}_{4} \mathrm{OS}+\mathrm{H} 577.1497$, found 577.1490.

(E)-1-((4-Methoxybenzylidene)amino)-5-(4-methoxyphenyl)-1'-propyl-2-thioxospiro[imidazolidine-4,3'-indolin]-2'-one (3af). Solid, mp 213-215 ${ }^{\circ}$; ${ }^{1} \mathrm{H}$ NMR (600 MHz, DMSO) $\delta 9.76$ $(\mathrm{s}, 1 \mathrm{H}), 7.87(\mathrm{~s}, 1 \mathrm{H}), 7.72(\mathrm{~d}, J 7.3 \mathrm{~Hz}, 1 \mathrm{H}), 7.43(\mathrm{td}, J 7.8,0.9 \mathrm{~Hz}, 1 \mathrm{H}), 7.31(\mathrm{t}, J 7.9 \mathrm{~Hz}, 1 \mathrm{H})$, $7.22(\mathrm{t}, J 7.5 \mathrm{~Hz}, 1 \mathrm{H}), 7.16-7.11(\mathrm{~m}, 3 \mathrm{H}), 7.03(\mathrm{~d}, J 7.9 \mathrm{~Hz}, 1 \mathrm{H}), 6.98(\mathrm{~d}, J$ 8.2, $2.2 \mathrm{~Hz}, 1 \mathrm{H}), 6.82$ $(\mathrm{d}, J 8.2,2.3 \mathrm{~Hz}, 1 \mathrm{H}), 6.47(\mathrm{~d}, J 7.7 \mathrm{~Hz}, 1 \mathrm{H}), 6.39(\mathrm{~s}, 1 \mathrm{H}), 5.81(\mathrm{~s}, 1 \mathrm{H}), 3.75(\mathrm{~s}, 3 \mathrm{H}), 3.57(\mathrm{~s}, 3 \mathrm{H})$, $3.23-3.14(\mathrm{~m}, 1 \mathrm{H}), 1.14-1.08(\mathrm{~m}, 1 \mathrm{H}), 1.06-1.00(\mathrm{~m}, 1 \mathrm{H}), 0.53(\mathrm{t}, J 7.4 \mathrm{~Hz}, 3 \mathrm{H}) .{ }^{13} \mathrm{C}$ NMR (150 MHz, DMSO) $\delta 183.2,172.3,159.9,159.4,149.5,143.9,135.7,133.56,131.1,130.3,129.8$, 127.3, 125.2, 123.4, 120.5, 119.6, 116.6, 114.2, 113.0, 112.0, 109.3, 72.1, 68.6, 55.6, 55.3, 41.2, 20.5, 11.2. ESI-HRMS: calcd for $\mathrm{C}_{28} \mathrm{H}_{28} \mathrm{~N}_{4} \mathrm{O}_{3} \mathrm{~S}+\mathrm{H}$ 501.1960, found 501.1963.

(E)-1-((3-Methoxybenzylidene)amino)-5-(3-methoxyphenyl)-1'-propyl-2-thioxospiro[imidazolidine-4,3'-indolin]-2'-one (3ag). Solid, mp 170-172 ${ }^{\circ} \mathrm{C} ;{ }^{1} \mathrm{H}$ NMR (600 MHz, DMSO) $\delta 9.76$ (s, 1H), $7.87(\mathrm{~s}, 1 \mathrm{H}), 7.72(\mathrm{~d}, J 7.3 \mathrm{~Hz}, 1 \mathrm{H}), 7.43(\mathrm{td}, J 7.8,0.9 \mathrm{~Hz}, 1 \mathrm{H}), 7.31(\mathrm{t}, J 7.9 \mathrm{~Hz}, 1 \mathrm{H})$, $7.22(\mathrm{t}, J 7.5 \mathrm{~Hz}, 1 \mathrm{H}), 7.16-7.11(\mathrm{~m}, 3 \mathrm{H}), 7.03(\mathrm{~d}, J 7.9 \mathrm{~Hz}, 1 \mathrm{H}), 6.98(\mathrm{~d}, J$ 8.2, $2.2 \mathrm{~Hz}, 1 \mathrm{H}), 6.82$ $(\mathrm{d}, J 8.2,2.3 \mathrm{~Hz}, 1 \mathrm{H}), 6.47(\mathrm{~d}, J 7.7 \mathrm{~Hz}, 1 \mathrm{H}), 6.39(\mathrm{~s}, 1 \mathrm{H}), 5.81(\mathrm{~s}, 1 \mathrm{H}), 3.75(\mathrm{~s}, 3 \mathrm{H}), 3.57(\mathrm{~s}, 3 \mathrm{H})$, $3.23-3.14(\mathrm{~m}, 1 \mathrm{H}), 1.14-1.08(\mathrm{~m}, 1 \mathrm{H}), 1.06-1.00(\mathrm{~m}, 1 \mathrm{H}), 0.53(\mathrm{t}, J 7.4 \mathrm{~Hz}, 3 \mathrm{H}) .{ }^{13} \mathrm{C} \mathrm{NMR}$ (150 MHz, DMSO) $\delta 183.2,172.3,159.9,159.4,149.5,143.9,135.7,133.5,131.1,130.3,129.8$, 127.3, 125.2, 123.4, 120.5, 119.6, 116.6, 114.2, 113.0, 112.0, 109.3, 72.1, 68.6, 55.6, 55.3, 41.2, 20.5, 11.2. ESI-HRMS: calcd for $\mathrm{C}_{28} \mathrm{H}_{28} \mathrm{~N}_{4} \mathrm{O}_{3} \mathrm{~S}+\mathrm{H}$ 501.1960, found 501.1963.

\section{(E)-5-(Furan-2-yl)-1-((furan-2-ylmethylene)amino)-1'-propyl-2-thioxospiro[imidazolidine-}

4,3'-indolin]-2' -one (3ah). Solid, mp 193-194 ${ }^{\circ} \mathrm{C} ;{ }^{1} \mathrm{H}$ NMR (600 MHz, DMSO) $\delta 9.75$ (s, 1H), $7.89(\mathrm{~s}, 1 \mathrm{H}), 7.83(\mathrm{~s}, 1 \mathrm{H}), 7.57(\mathrm{~s}, 1 \mathrm{H}), 7.41(\mathrm{t}, J 7.8 \mathrm{~Hz}, 1 \mathrm{H}), 7.17(\mathrm{t}, J 7.5 \mathrm{~Hz}, 1 \mathrm{H}), 7.09$ (d, J 7.9 $\mathrm{Hz}, 1 \mathrm{H}), 6.82(\mathrm{~d}, J 3.4 \mathrm{~Hz}, 1 \mathrm{H}), 6.61(\mathrm{dd}, J 3.2,1.7 \mathrm{~Hz}, 1 \mathrm{H}), 6.41-6.37(\mathrm{~m}, 1 \mathrm{H}), 6.24(\mathrm{~d}, J 3.3$ $\mathrm{Hz}, 1 \mathrm{H}), 5.97(\mathrm{~s}, 1 \mathrm{H}), 3.57(\mathrm{~m}, J 13.9,7.0 \mathrm{~Hz}, 1 \mathrm{H}), 3.39-3.35(\mathrm{~m}, 1 \mathrm{H}), 2.51(\mathrm{~s}, 1 \mathrm{H}), 1.40(\mathrm{~m}, J$ $31.7,13.8,7.0 \mathrm{~Hz}, 2 \mathrm{H}), 0.74(\mathrm{t}, J 7.4 \mathrm{~Hz}, 3 \mathrm{H}) .{ }^{13} \mathrm{C} \mathrm{NMR}(150 \mathrm{MHz}, \mathrm{DMSO}) \delta 181.8,171.9,149.3$, $145.8,144.1,143.4,139.2$, 131.0, 128.0, 124.7, 123.4, 114.7, 112.7, 111.3, 110.9, 109.5, 67.2, 66.1, 41.4, 20.6, 11.4. ESI-HRMS: calcd for $\mathrm{C}_{22} \mathrm{H}_{20} \mathrm{~N}_{4} \mathrm{O}_{3} \mathrm{~S}+\mathrm{H} 421.1334$, found 421.1329 .

(E)-1-((2-Chlorobenzylidene)amino)-5-(2-chlorophenyl)-1'-methyl-2-thioxospiro[imidazolidine-4,3'-indolin]-2'-one (3bi). White solid (432.1 mg, 90\% yield), mp 201-203 ${ }^{\circ} \mathrm{C}$; ${ }^{1} \mathrm{H}$ NMR (600 MHz, DMSO) $\delta 9.96(\mathrm{~s}, 1 \mathrm{H}), 7.97$ (d, J 6.9 Hz, 1H), 7.64 (d, J 7.4 Hz, 1H), 7.58 (s, 1H), 7.46 - 7.37 (m, 5H), 7.35 - 7.30 (m, 2H), 7.17 (t, J 11.0, 4.1 Hz, 1H), 7.06 (m, J 14.4, 6.9, 5.2 Hz, 2H), $6.19(\mathrm{~s}, 1 \mathrm{H}), 2.91$ (s, 3H). ${ }^{13} \mathrm{C}$ NMR (150 MHz, DMSO) $\delta 181.6,171.4,133.5,132.3,131.9,131.5$, 
$131.1,130.9,130.3,130.1,130.0,129.0,128.1,127.6,127.2,124.7,123.8,109.4,100.0,67.7$, 26.7. ESI-HRMS: calcd for $\mathrm{C}_{24} \mathrm{H}_{18} \mathrm{Cl}_{2} \mathrm{~N}_{4} \mathrm{OS}+\mathrm{H} 481.0657$, found 481.0651 .

(E)-1'-Methyl-1-((4-nitrobenzylidene)amino)-5-(4-nitrophenyl)-2-thioxospiro[imidazolidine-4,3'-indolin]-2'-one (3bj). Yellow solid (446.8 mg, 89\% yield), mp 211-213 ${ }^{\circ} \mathrm{C}$; ${ }^{1} \mathrm{H} \mathrm{NMR}$ (600 MHz, DMSO) $\delta 10.01(\mathrm{~s}, 1 \mathrm{H}), 8.40(\mathrm{~s}, 1 \mathrm{H}), 8.22-8.19(\mathrm{~m}, 1 \mathrm{H}), 8.17(\mathrm{~d}, \mathrm{~J} 8.3,1.6 \mathrm{~Hz}, 1 \mathrm{H})$, 8.01 (d, J 7.9 Hz, 1H), 7.92 (s, 1H), 7.71 (t, J 6.5 Hz, 2H), 7.61 (t, J 8.0 Hz, 1H), 7.49 (t, J 11.3, $4.3 \mathrm{~Hz}, 1 \mathrm{H}), 7.36$ (d, J $7.8 \mathrm{~Hz}, 1 \mathrm{H}), 7.26$ (t, J $7.5 \mathrm{~Hz}, 1 \mathrm{H}), 7.06$ (d, J $7.9 \mathrm{~Hz}, 1 \mathrm{H}), 6.12$ (s, 1H), 2.79 (s, 3H). ${ }^{13} \mathrm{C}$ NMR (150 MHz, DMSO) $\delta 182.7,171.9,148.6,147.9,145.2,143.7,136.2,134.3$, 134.0, 133.5, 131.5, 130.9, 130.6, 127.6, 124.9, 124.3, 124.1, 122.0, 121.8, 109.7, 70.2, 68.4, 26.6. ESI-HRMS: calcd for $\mathrm{C}_{24} \mathrm{H}_{18} \mathrm{~N}_{6} \mathrm{O}_{5} \mathrm{~S}+\mathrm{H}$ 503.1059, found 503.1119.

(E)-1'-Methyl-1-((3-nitrobenzylidene)amino)-5-(3-nitrophenyl)-2-thioxospiro[imidazolidine-4,3'-indolin]-2'-one (3bk). White solid (426.8 mg, 85\% yield), mp 207-209 ${ }^{\circ} \mathrm{C}$; ${ }^{1} \mathrm{H}$ NMR (600 MHz, DMSO) $\delta 10.03(\mathrm{~s}, 1 \mathrm{H}), 8.41(\mathrm{~s}, 1 \mathrm{H}), 8.20(\mathrm{dd}, \mathrm{J} 23.6,8.3 \mathrm{~Hz}, 2 \mathrm{H}), 8.03$ (d, J 7.9 Hz, $1 \mathrm{H}), 7.93(\mathrm{~s}, 1 \mathrm{H}), 7.74-7.67(\mathrm{~m}, 3 \mathrm{H}), 7.62(\mathrm{t}, J 8.0 \mathrm{~Hz}, 1 \mathrm{H}), 7.51(\mathrm{t}, J 7.7 \mathrm{~Hz}, 1 \mathrm{H}), 7.37(\mathrm{~d}, J 7.7$ $\mathrm{Hz}, 1 \mathrm{H}), 7.27(\mathrm{t}, J 7.5 \mathrm{~Hz}, 1 \mathrm{H}), 7.07(\mathrm{~d}, J 7.9 \mathrm{~Hz}, 1 \mathrm{H}), 6.13(\mathrm{~s}, 1 \mathrm{H}), 2.81(\mathrm{~s}, 3 \mathrm{H}) .{ }^{13} \mathrm{C}$ NMR $(150$ MHz, DMSO) $\delta 182.7,171.9,148.6,147.9,145.3,143.7,136.2,134.3,134.0,133.5,131.5,130.9$, 130.6, 127.6, 124.9, 124.3, 124.1, 122.0, 121.8, 109.7, 68.4, 26.6. ESI-HRMS: calcd for $\mathrm{C}_{24} \mathrm{H}_{18} \mathrm{~N}_{6} \mathrm{O}_{5} \mathrm{~S}+\mathrm{H}$ 503.1059, found 503.1125.

(E)-1'-Methyl-1-((2-methylbenzylidene)amino)-2-thioxo-5-(o-tolyl)spiro[imidazolidine-4,3'indolin]-2'-one (3bl). White solid (299.3 mg, 68\% yield), $\mathrm{mp}=183-185{ }^{\circ} \mathrm{C} ;{ }^{1} \mathrm{H} \mathrm{NMR}(600 \mathrm{MHz}$, DMSO) $\delta 9.66(\mathrm{~s}, 1 \mathrm{H}), 7.73-7.71(\mathrm{~m}, 1 \mathrm{H}), 7.69(\mathrm{~d}, J 7.3 \mathrm{~Hz}, 1 \mathrm{H}), 7.52(\mathrm{~s}, 1 \mathrm{H}), 7.46-7.42(\mathrm{~m}$, $1 \mathrm{H}), 7.23-7.18(\mathrm{~m}, 3 \mathrm{H}), 7.14-7.11(\mathrm{~m}, 2 \mathrm{H}), 7.09-7.06(\mathrm{~m}, 2 \mathrm{H}), 7.00(\mathrm{~d}, J 7.8 \mathrm{~Hz}, 1 \mathrm{H}), 6.14$ $(\mathrm{s}, 1 \mathrm{H}), 2.82(\mathrm{~s}, 3 \mathrm{H}), 1.87(\mathrm{~s}, 3 \mathrm{H}), 1.72(\mathrm{~s}, 3 \mathrm{H}) .{ }^{13} \mathrm{C}$ NMR (150 MHz, DMSO) $\delta 182.3,172.5$, 144.9, 143.9, 137.0, 135.9, 132.3, 131.1, 130.9, 130.1, 129.7, 128.8, 128.6, 126.6, 126.1, 125.0, 123.7, 109.4, 68.3, 26.6, 18.7. ESI-HRMS: calcd for $\mathrm{C}_{26} \mathrm{H}_{24} \mathrm{~N}_{4} \mathrm{OS}+\mathrm{H} 441.1671$, found 441.1739. (E)-1'-Methyl-1-((3-methylbenzylidene)amino)-2-thioxo-5-(m-tolyl)spiro[imidazolidine-

4,3'-indolin]-2' -one (3bm). White solid (286.1 mg, 65\% yield), mp = 179-180 ${ }^{\circ} \mathrm{C} ;{ }^{1} \mathrm{H}$ NMR (600 $\mathrm{MHz}, \mathrm{CDCl} 3) \delta 7.94(\mathrm{~s}, 1 \mathrm{H}), 7.65(\mathrm{~d}, J 7.3 \mathrm{~Hz}, 1 \mathrm{H}), 7.49(\mathrm{~s}, 1 \mathrm{H}), 7.44(\mathrm{t}, J 7.5 \mathrm{~Hz}, 1 \mathrm{H}), 7.39$ (d, $J 7.6 \mathrm{~Hz}, 1 \mathrm{H}), 7.25-7.20(\mathrm{~m}, 2 \mathrm{H}), 7.18(\mathrm{~d}, J 4.5 \mathrm{~Hz}, 1 \mathrm{H}), 7.12(\mathrm{t}, J 7.5 \mathrm{~Hz}, 1 \mathrm{H}), 7.07$ (d, J 7.6 Hz, $1 \mathrm{H}), 6.78(\mathrm{~d}, J 8.6 \mathrm{~Hz}, 2 \mathrm{H}), 5.52(\mathrm{~s}, 1 \mathrm{H}), 2.82(\mathrm{~s}, 3 \mathrm{H}), 2.33(\mathrm{~s}, 3 \mathrm{H}), 2.23(\mathrm{~s}, 3 \mathrm{H}) .{ }^{13} \mathrm{C}$ NMR $(150$ $\mathrm{MHz}, \mathrm{CDCl} 3) \delta 183.4,172.2,151.1,143.7,138.3,133.6,131.3,131.1,129.8,128.4,128.1,127.4$, 127.3, 125.3, 124.4, 123.9, 123.8, 108.6, 68.7, 26.2, 21.3. ESI-HRMS: calcd for $\mathrm{C}_{26} \mathrm{H}_{24} \mathrm{~N}_{4} \mathrm{OS}+\mathrm{H}$ 441.1671 , found 441.1742 .

\section{Acknowledgements}

We are grateful for the financial support from the National Natural Science Foundation of P. R. of China (No. 21272214, 21502172), Zhejiang Provincial Natural Science Foundation of China (LQ13B020005) and Ningbo Natural Science Foundation (2015A610284). 


\section{References}

1. Rousseau, G.; Robert, F.; Schenk, K.; Landais, Y. Org. Lett. 2008, 10, 4441. http://pubs.acs.org/doi/abs/10.1021/ol8016885

2. Zhao, F.; Wang, C.; Liu, L.; Zhang, W.-X.; Xi, Z. Chem. Commun. 2009, 6569. http://pubs.rsc.org/en/content/articlelanding/2009/cc/b914619a

3. Murai, K.; Komatsu, H.; Nagao, R.; Fujioka, H. Org. Lett. 2012, 14, 772. http://pubs.acs.org/doi/abs/10.1021/ol203313n

4. Bogdanowicz-Szwed, K.; Budzowski, A.; Gil, R.; Serda, P. Monatsh. Chem. 2010, 141, 63. http://link.springer.com/article/10.1007/s00706-009-0233-4

5. Liu, X.-L.; Han, W.-Y.; Zhang, X.-M.; Yuan, W.-C. Ogr. Lett. 2013, 15, 1246. http://pubs.acs.org/doi/abs/10.1021/ol400183k

6. Han, W.-Y.; Li, S.-W.; Wu, Z.-J.; Zhang, X.-M.; Yuan, W.-C. Chem. Eur. J. 2013, 19, 5551. http://onlinelibrary.wiley.com/doi/10.1002/chem.201300206/full

7. Cao, Y.-M.; Shen, F.-F.; Zhang, F.-T.; Wang, R. Chem. Eur. J. 2013, 19, 1184. http://onlinelibrary.wiley.com/doi/10.1002/chem.201204114/full

8. Wu, H.; Zhang, L.-L.; Tian, Z.-Q.; Huang, Y.-D.; Wang, Y.-M. Chem. Eur. J. 2013, 19, 1747. http://onlinelibrary.wiley.com/doi/10.1002/chem.201203221/full

9. Tan, F.; Cheng, H-G.; Feng, B.; Zou, Y-Q.; Duan, S.-W.; Chen, J.-R.; Xiao, W.-J. Eur. J. Org. Chem. 2013, 2071.

http://onlinelibrary.wiley.com/doi/10.1002/ejoc.201300081/full

10. Han, Y.-Y.; Chen, W.-B.; Han, W.-Y.; Wu, Z.-J.; Zhang, X.-M.; Yuan, W.-C. Ogr. Lett. 2012, $14,490$.

http://pubs.acs.org/doi/abs/10.1021/ol203081x

11. Kato, S.; Yoshino, T.; Shibasaki, M.; Kanai, M.; Matsunaga, S. Angew. Chem. Int. Ed. 2012, $51,7007$. http://onlinelibrary.wiley.com/doi/10.1002/anie.201203005/full

12. Chen, W.-B.; Wu, Z.-J.; Hu, J.; Cun, L.-F.; Zhang, X.-M.; Yuan, W.-C. Ogr. Lett. 2011, 13, 2472.

http://pubs.acs.org/doi/abs/10.1021/ol200724q

13. Chen, W.-B.; Han, W.-Y.; Han, Y.-Y.; Zhang, X.-M.; Yuan, W.-C. Tetrahedron 2013, 69, 5281.

http://www.sciencedirect.com/science/article/pii/S004040201300714X

14. Du, D.; Jiang, Y.; Xu, Q.; Shi, M. Adv. Synth. Catal. 2013, 355, 2249.

http://onlinelibrary.wiley.com/doi/10.1002/adsc.201300460/full

15. Zhang, Y. L.; Chen, B. Z.; Zheng, K. Q.; Xu, M. L.; Lei, X. H. Yao Xue Bao 1982, 17, 17. http://www.yxxb.com.cn:8081/aps/CN/abstract/abstract12979.shtml

16. Bonsignore, L.; Loy, G.; Secci, D.; Calignano, A. Eur. J. Med. Chem., 1993, 28, 517. http://www.sciencedirect.com/science/article/pii/022352349390020F 
17. Müller-Vahl, K. R.; Schneider, U.; Koblenz, A.; Jöbges, M.; Kolbe, H.; Daldrup, T.; Emrich, H. M. Pharmacopsychiatry, 2002, 35, 57.

https://www.thieme-connect.com/products/ejournals/abstract/10.1055/s-2002-25028

18. Müller-Vahl, K. R.; Prevedel, H.; Theloe, K.; Kolbe, H.; Emrich, H. M.; Schneider, U. Neuropsychopharmacology 2003, 28, 384.

http://www.nature.com/npp/journal/v28/n2/full/1300047a.html

19. Fu, Z.-K.; Pan, J.-Y.; Xu, D.-C.; Xie, J.-W. RSC Advances, 2014, 4, 51548.

http://pubs.rsc.org/en/content/articlelanding/2014/ra/c4ra07860h\#!divAbstract 